\title{
Reduced synthesis of the Ybt siderophore or production of aberrant Ybt-like molecules activates transcription of yersiniabactin genes in Yersinia pestis
}

Correspondence

Robert D. Perry

rperry@uky.edu
Received 6 January 2010

Revised 24 March 2010

Accepted 20 April 2010

\author{
M. Clarke Miller, ${ }^{1} \dagger$ Jacqueline D. Fetherston, ${ }^{2}$ Carol L. Pickett, ${ }^{2}$ \\ Alexander G. Bobrov, ${ }^{2}$ Robert H. Weaver, ${ }^{2} \ddagger$ Edward DeMoll ${ }^{3}$ \\ and Robert D. Perry ${ }^{2}$ \\ ${ }^{1}$ Department of Chemistry, University of Kentucky, Lexington, KY 40506-0055, USA \\ ${ }^{2}$ Department of Microbiology, Immunology, and Molecular Genetics, University of Kentucky, 800 \\ Rose St. MS-415 Medical Center, Lexington, KY 40536-0298, USA \\ ${ }^{3}$ Department of Biology, 101 T. H. Morgan Building, Lexington, KY 40506-0225, USA
}

\section{INTRODUCTION}

Iron is essential for nearly all pathogenic bacteria as well as for their hosts. Under normal conditions, the host binds iron to specific proteins, making free iron essentially unavailable to invading pathogens. Consequently, obtaining iron from these host proteins is one prerequisite for most pathogens to cause infection and disease (Braun, 2001, 2005; Bullen et al., 2005; Schaible \& Kaufmann, 2004). Yersinia pestis, the causative agent of bubonic and pneumonic plague, encodes a large number of proven or

tPresent address: James Graham Brown Cancer Center, University of Louisville, 529 S. Jackson Street, Louisville, KY 40202, USA.

‡Present address: University of Kentucky College of Medicine, 138 Leader Ave., Lexington, Kentucky, 40506-9983, USA.

Abbreviations: CR, Congo red; DIP, 2,2'-dipyridyl; HPI, high-pathogenicity island; NRPS, nonribosomal peptide synthetase; PKS, polyketide synthase; TE, thioesterase. putative iron-transport systems. Of these, the siderophoredependent yersiniabactin (Ybt) biosynthesis and transport system has proven to be essential for the ability of the organism to cause bubonic plague.

Except for $y b t D$ and fur, all identified genes required for the synthesis and transport of Ybt and the regulation of the entire system are encoded within a high-pathogenicity island (HPI) that is found in a number of enteric pathogens, including Yersinia enterocolitica and Yersinia pseudotuberculosis (Bobrov et al., 2002; Deng et al., 2002; Lesic \& Carniel, 2004; Perry \& Fetherston, 2004). Ybt synthesis occurs by a mixed nonribosomal peptide synthetase (NRPS)/polyketide synthase (PKS) mechanism (Fig. 1a). Salicylate, three cysteines, a malonyl linker group and three methyl groups are assembled into a four-ring structure made of salicylate, one thiazolidine, and two thiazoline rings with a malonyl linker separating the final thiazoline from the thiazolidine ring (Chambers et al., 


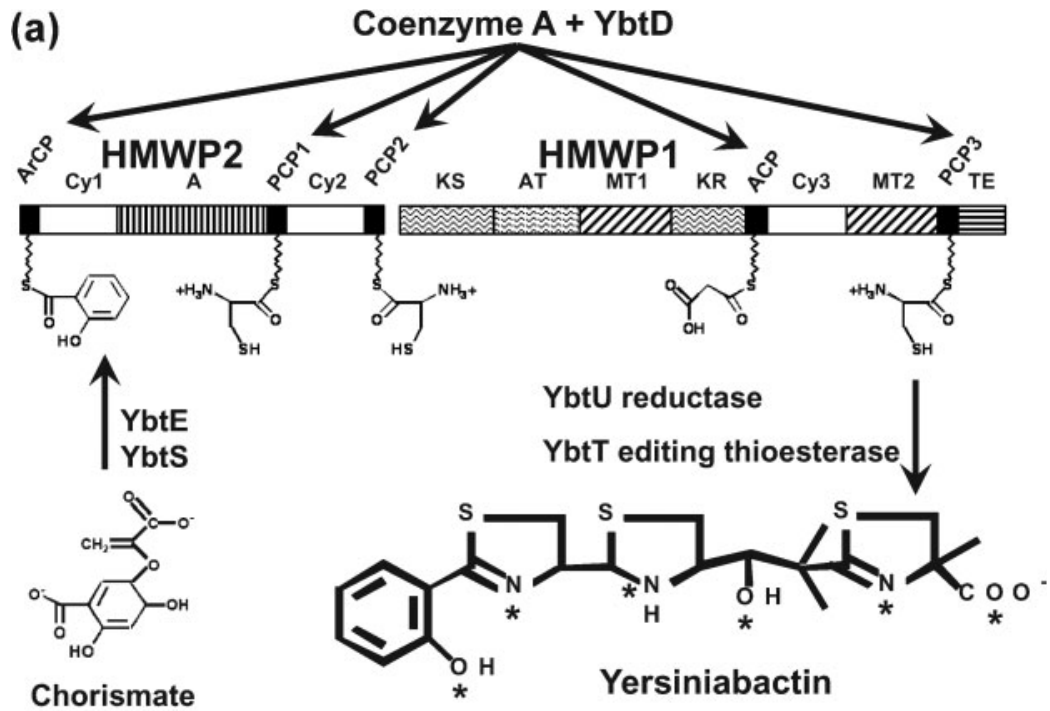

(b)

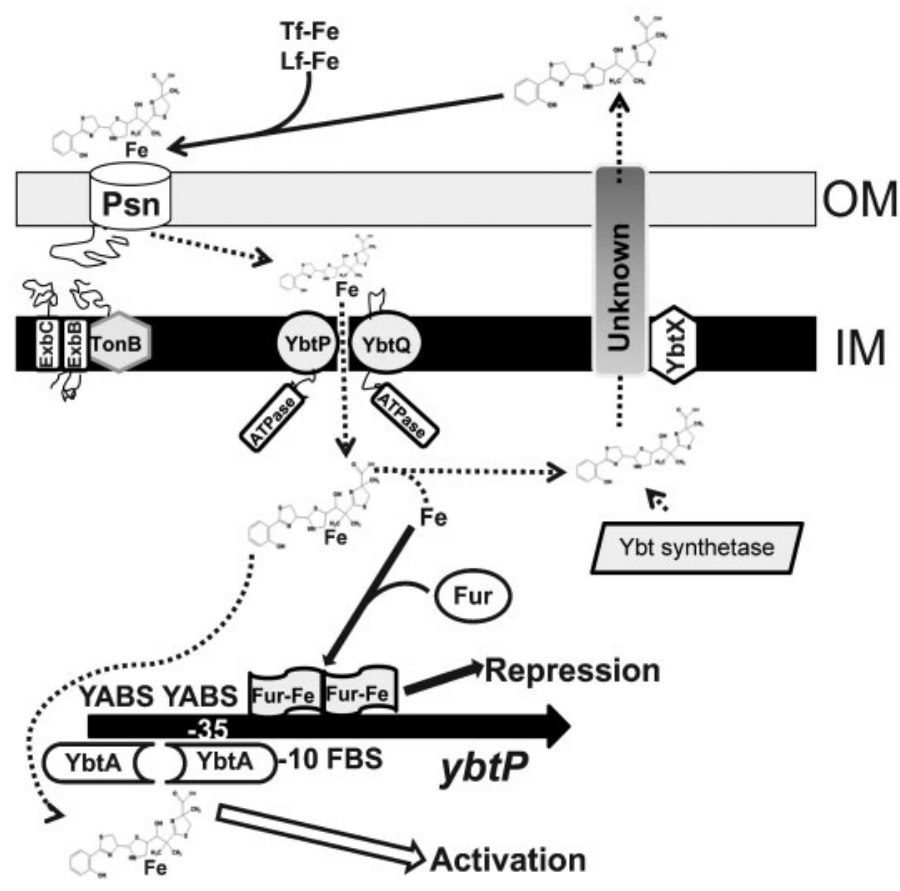

Fig. 1. Models of yersiniabactin (Ybt) biosynthesis, transport and gene regulation. (a) Ybt biosynthesis. YbtS converts chorismate to salicylate and YbtE adenylates salicylate for attachment to HMWP2. YbtD transfers phosphopantetheinyl groups from coenzyme $A$ to the indicated sites on HMWP2 and HMWP1 for attachment of salicylate, three cysteines and malonate. YbtU reduces one thiazolidine ring to a thiazoline ring, while YbtT is a TE that likely removes aberrant molecules from the Ybt synthetase enzyme complex. The TE domain of HMWP1 likely releases the completed siderophore from the enzyme complex. Asterisks in the Ybt structure indicate ferric ion coordination sites. HMWP2 and HMWP1 enzymatic domains: ArCP, aryl carrier protein; $\mathrm{Cy}$, condensation/cyclization; A, adenylation; PCP, peptidyl carrier protein; KS, ketoacyl synthase; AT, acyltransferase; MT, methyltransferase; $\mathrm{KR}, \quad \beta$-ketoreductase; $\mathrm{ACP}, \quad$ acyl carrier protein; TE, thioesterase. The model in (a) is reproduced with modifications from Perry \& Fetherston (2004) with the permission of Horizon Scientific Press/Caister Academic Press, UK. (b) Model of Ybt transport and transcriptional regulation of $y b t P$. Synthesized Ybt is exported via an unknown mechanism that may include YbtX. Secreted Ybt can remove ferric iron from transferrin (Tf-Fe) and lactoferrin ( $\mathrm{Lf}-\mathrm{Fe})$. We propose that the $\mathrm{Ybt}-$ Fe complex is transported through the outer membrane via the TonB-dependent receptor Psn. Ybt-Fe is transported through the inner membrane by $\mathrm{YbtP} / \mathrm{YbtQ}$. It is unclear whether this step requires a periplasmic or a membrane-spanning protein (none is shown here). In the cytoplasm, iron is released from Ybt by an unknown mechanism and is used nutritionally and for regulation of gene expression. The ybtP promoter, which controls expression of the $y b t P Q X S$ operon, is activated by $\mathrm{YbtA}$, probably complexed with $\mathrm{Ybt}$, and repressed by Fur in the presence of excess iron. Promoters for the irp2-irp1-ybtUTE and psn operons are similarly regulated. In contrast, YbtA negatively regulates transcription from its own promoter (Perry, 2004; Perry \& Fetherston, 2004). YABS, YbtA binding site; FBS, Fur binding site; promoter region indicated by -10 and -35 . Dashed arrows indicate steps or transported substrates that have not been experimentally determined.
1996; Crosa \& Walsh, 2002; Drechsel et al., 1995; Gehring et al., 1998a; Miethke \& Marahiel, 2007; Perry et al., 1999). YbtD is a putative phosphopantetheinyl (P-pant) transferase responsible for adding the phosphopantetheine tethers for the cysteine, salicylate and malonyl groups to HMWP1 and HMWP2. YbtS synthesizes salicylate from chorismate, which is then adenylated by $\mathrm{YbtE}$ and transferred to the HMWP2-HMWP1 assembly complex. Two cysteines are cyclized and condensed to form two thiazoline rings on HMWP2 (encoded by irp2). A malonyl linker is added and 
$\mathrm{YbtU}$ reduces the second thiazoline ring to thiazolidine before cyclization and condensation of the final thiazoline ring on HMWP1 (encoded by irp1). The YbtT putative thioesterase (TE) likely serves an editing function to remove aberrant molecules from the enzyme complex, while the TE domain of HMWP1 likely releases the completed siderophore from the enzyme complex (Fig. 1a) (Crosa \& Walsh, 2002; Gehring et al., 1998a; Geoffroy et al., 2000; Kerbarh et al., 2005; Miller et al., 2002; Pelludat et al., 2003). The formation constant of this siderophore with ferric iron is $4 \times 10^{36}$ and the crystal structure of the complex has been solved (Miller et al., 2006; Perry et al., 1999).

YbtX is likely involved in, but not essential for, Ybt export from the bacterial cell (Fig. 1b). Use of iron from the Ybt-Fe complex requires the TonB-dependent outer membrane receptor Psn (termed FyuA in Y. enterocolitica) and an ABC transporter system consisting of YbtP and YbtQ: two fusedfunction inner membrane proteins with both permease and ATPase domains (Fig. 1b). Most ABC uptake transporters have separate polypeptides that encode permease and ATPase domains, while proteins that contain both a permease and an ATPase are usually involved in export. However, it has been demonstrated that YbtP and YbtQ are both required for uptake and are not essential for siderophore export (Fetherston et al., 1995, 1999; Perry et al., 2003b).

The four $y b t$ operons within the HPI, (1) psn, (2) irp2-irp1$y b t U-y b t T-y b t E$, (3) $y b t A$ and (4) $y b t P-y b t Q-y b t X-y b t S$, are repressed by the Fur-Fe complex. In addition to Fur and iron repression, expression of the Ybt system is controlled by $\mathrm{YbtA}$, a member of the AraC family of transcriptional regulators, and the Ybt siderophore. Together, YbtA and the Ybt siderophore activate transcription from the $p s n$, irp2 and $y b t P$ promoters while repressing transcription of the $y b t A$ promoter (Anisimov et al., 2005; Fetherston et al., 1996, 1999; Gehring et al., 1998a; Perry et al., 2003a; Staggs et al., 1994). Repeats within the promoter regions of the $y b t$ genes are proposed as YbtA binding sites. Mutation of the repeat in the psn promoter causes loss of transcriptional activation (Fetherston et al., 1996, 1999; Perry et al., 2003a, b). In Y. enterocolitica, Anisimov et al. (2005) have demonstrated that YbtA binds to these repeats in the irp6 $(y b t P)$ promoter. Ybt is a potent signal molecule: concentrations 500-fold lower than that required for growth stimulation activate the $y b t P$ promoter (Perry et al., 2003a).

A mutation in any of the seven genes encoding Ybtbiosynthetic enzymes causes a loss of siderophore production, as assessed by growth stimulation in bioassays. The $y b t D, y b t E, y b t U$, irp1 and $i r p 2$ mutations each cause a loss of Ybt protein expression due to lower transcription from the $p s n$, irp2 and $y b t P$ promoters. Addition of purified Ybt to Ybt-biosynthetic mutants restores YbtA-dependent transcriptional activation (Perry et al., 2003a). An unexpected finding was that mutations in $y b t S, y b t T$ and the TE domain of irp1 (irp1-2086) do not affect regulation of the Ybt system (Bearden et al., 1997; Bobrov et al., 2002; Fetherston et al., 1995, 1996; Geoffroy et al., 2000; Perry et al., 2003a).
In this study we examined the means by which $y b t S, y b t T$ and irp1-2086 mutants maintain normal regulation of the Ybt system. We found that the TE mutants $y b t T$ and irp12086 produce reduced amounts of authentic Ybt. Although the $y b t S$ mutant does not make Ybt, this mutant and a chromosomal deletion mutant lacking the entire HPI (a $\Delta p g m$ strain) make Ybt-like molecules capable of transcriptionally activating the $y b t P$ promoter. Finally, we show that substitutions in $\mathrm{YbtT}$ residues found to be critical for TE activity in other type II TEs cause a phenotype similar to that of a $y b t T$ mutant.

\section{METHODS}

Bacterial strains and cultivation. All bacterial strains and plasmids used in this study are described in Table 1. All Y. pestis strains used were avirulent due to lack of the virulence plasmid pCD1 (Perry \& Fetherston, 1997; Perry et al., 1998). Cells from buffered glycerol stocks (Beesley et al., 1967) stored at $-80{ }^{\circ} \mathrm{C}$ were streaked onto Congo red (CR) plates (Surgalla \& Beesley, 1969) and incubated at $30{ }^{\circ} \mathrm{C}$ for $48 \mathrm{~h}$ to confirm retention of the pgm locus. ORFs for the Ybt system are encoded on the HPI within the pgm locus (Lesic \& Carniel, 2004; Perry \& Fetherston, 2004). $\mathrm{CR}^{+}$colonies were inoculated on Tryptose Blood Agar Base (TBA; Difco Laboratories) slants and incubated at $30{ }^{\circ} \mathrm{C}$ for 24-48 h. Cells were washed off TBA slants with deferrated PMH2 medium (Gong et al., 2001), inoculated to an $\mathrm{OD}_{620}$ of 0.1 , and incubated for $\sim 8 \mathrm{~h}$ at $37{ }^{\circ} \mathrm{C}$ with aeration in a New Brunswick model G76 gyratory shaker water bath (200 r.p.m.). Gong et al. (2001) has an error in the published buffer concentrations: PIPES and HEPES should be $50 \mathrm{mM}$ for $\mathrm{PMH} 2$ and $\mathrm{PMH}$, respectively. For siderophore isolation and bioassays, cultures were diluted into fresh, deferrated $\mathrm{PMH}_{2}$ to $\mathrm{OD}_{620} 0.1$ and incubated at $37{ }^{\circ} \mathrm{C}$ for $\sim 16 \mathrm{~h}$. Both the $\mathrm{Ybt}^{+}$strain and Ybt-biosynthetic mutants have similar growth rates and similar cell yields under these growth conditions (Fetherston et al., 2010). Thus, culture supernatants for Ybt isolation or direct use in regulation studies are from cultures incubated for the same time periods and harvested from similar cell yields. Cells were removed from spent $\mathrm{PMH} 2$ by centrifugation and filtration of the supernatant through $0.22 \mu \mathrm{m}$ pore-size filters. Cell growth in liquid cultures was monitored with a Genesys 5 spectrophotometer (Spectronic Instruments). All glassware used for iron-restricted studies was soaked overnight in 5\% Micro-90 (ColeParmer) to remove contaminating iron and copiously rinsed in deionized water.

Construction of $\boldsymbol{Y}$. pestis mutant and reporter strains. A deletion in $y 2336$, which encodes a protein with similarity to $\mathrm{YbtS}$, was constructed, leaving the first $150 \mathrm{bp}$ and last $44 \mathrm{bp}$ of the gene. Total genomic DNA from KIM10 + was digested with HindIII and SalI and separated by agrose gel electrophoresis. Fragments in the 3-4 kb range were eluted from the low-temperature-melting agarose and ligated into the HindIII-SalI sites of pTrueBlue-rop. Clones with the $3.4 \mathrm{~kb}$ HindIII-SalI fragment carrying y2336 were detected by PCR using primers IHS-1 and IHS-2. PCR results were confirmed by restriction analysis, and one recombinant plasmid was named pIHS1. A 1777 bp deletion was made in $y 2336$ by eliminating the NruI-StuI internal fragment, creating pIHS2. A $1.6 \mathrm{~kb}$ HindIII-Sall fragment from pIHS2 was cloned into pWSK29 to yield pIHS3, and the SalI-XbaI fragment from this plasmid was cloned into pKNG101, generating the suicide vector pKNGY $\Delta 2336$. The $y 2336$ deletion was introduced into KIM6+ and KIM6-2070.1 ( $y b t S:$ : kan2070.1) by allelic exchange, as previously described (Fetherston et al., 1999). A Y. pestis mutant carrying the y2336 deletion in the chromosome (KIM6-2070.2) was verified by PCR with primers IHS-1, IHS-2 and IHS-3 (Table 2). Clones which did not 
Table 1. Bacterial strains and plasmids used in this study

All Y. pestis strains are $\mathrm{Lcr}^{-}$and thus avirulent due to a lack of the low-calcium-response plasmid pCD1. Strains with a plus sign possess an intact $102 \mathrm{~kb}$ pgm locus containing the genes for biofilm formation $(\mathrm{hms})$ and the Ybt iron-transport system. All other Y. pestis strains have either a pgm deletion or a mutation within the pgm locus. Abbreviations: $\mathrm{Ap}^{\mathrm{r}}, \mathrm{Cm}^{\mathrm{r}}, \mathrm{Km}^{\mathrm{r}}, \mathrm{Spc}^{\mathrm{r}}$ and $\mathrm{Sm}^{\mathrm{r}}$, respectively indicate resistance to ampicillin, chloramphenicol, kanamycin, spectinomycin and streptomycin.

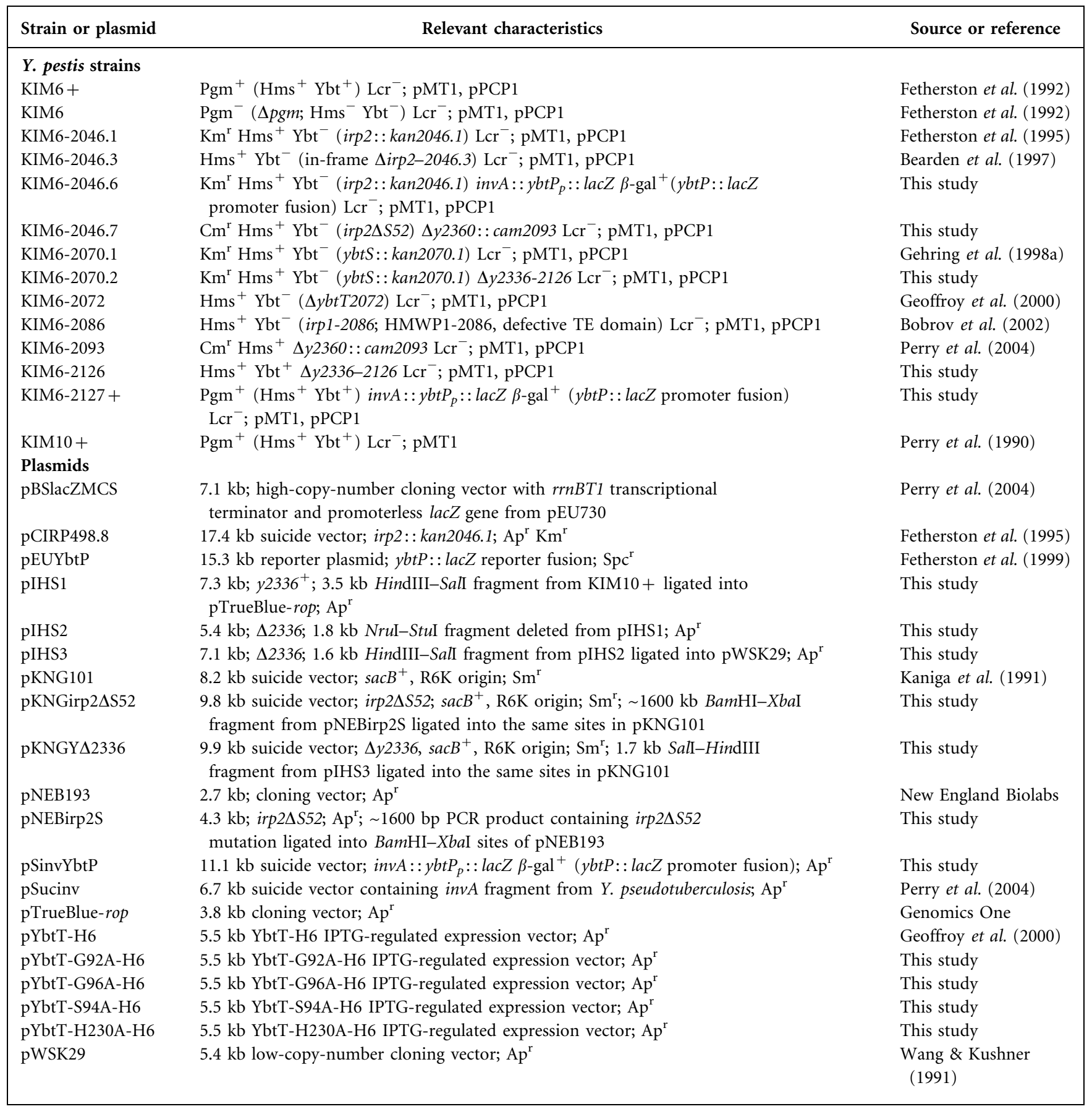

show any PCR product with IHS-1 and IHS-3 (which lies inside the deleted region) were confirmed by PCR with IHS-1 and IHS-2. The resulting strains were designated KIM6-2126 ( $\left.y b t S^{+} \Delta y 2336-2126\right)$ and KIM6-2070.2 (ybtS: : kan2070.1 $\Delta y 2336-2126)$.

The 166 bp $y b t P$ promoter region was amplified from pEUYbtP (Fetherston et al., 1999) using primers YbtP-2 and YbtA-2 (Table 2).
The PCR products were digested with Asp718 and cloned into pBSlacZMCS (Perry et al., 2004) that had been digested with Asp718 and treated with alkaline phosphatase. Clones containing the lac $Z$ gene under the control of the $y b t P$ promoter were identified by sequence analysis. An $\sim 4.4 \mathrm{~kb}$ EagI fragment was isolated from this clone and ligated into the EagI site of pSucinv (Perry et al., 2004) to generate $\mathrm{pSinvYbtP}$. The $y b t P::$ lac $Z$ reporter was integrated into the 
Table 2. Primers used in this study

\begin{tabular}{|c|c|c|}
\hline Primer & Sequence $\left(5^{\prime}-3^{\prime}\right)$ & Purpose \\
\hline$\Delta \operatorname{irpS}$ & CCAGGCCGGCCTGGATTCC & Check irp2 $\Delta S 52$ deletion \\
\hline G92A forward & GCTTTTACTCGCCGCGCACAGCATGGGGG & Construction YbtT-G92A-H6 \\
\hline G92A reverse & CCCCCATGCTGTGCGCGGCGAGTAAAAGC & Construction YbtT-G92A-H6 \\
\hline G96A forward & CGGGCACAGCATGGCGGCGCAGGTGGCG & Construction YbtT-G96A-H6 \\
\hline G96A reverse & CGCCACCTGCGCCGCCATGCTGTGCCCG & Construction YbtT-G96A-H6 \\
\hline S94A forward & CGCCGGGCACGCCATGGGGGCGCAGG & Construction YbtT-S94A-H6 \\
\hline S94A reverse & CCTGCGCCCCCATGGCGTGCCCGGCG & Construction YbtT-S94A-H6 \\
\hline H230A forward & GACGGCGATGCTTTCTATCCCATTCAACAAGC & Construction YbtT-H230A-H6 \\
\hline $\mathrm{H} 230 \mathrm{~A}$ reverse & GCTTGTTGAATGGGATAGAAAGCATCGCCGTC & Construction YbtT-H230A-H6 \\
\hline IHS-1 & AGAAACTGCCGAAATGGTGAGG & $\begin{array}{l}\text { Clone } y 2336 \text { and check } y 2336 \\
\text { deletion }\end{array}$ \\
\hline IHS-2 & GAGAGGGAAGCAAACTGCGTATT & Clone $y 2336$ \\
\hline IHS-3 & TTCCCTGGAGATGTTCCCGT & Check $y 2336$ deletion \\
\hline S1 & CGGGATCCGCATGCACGGCGGCTTCAG & Construct $\operatorname{irp} 2 \Delta S 52$ deletion \\
\hline S2 & ATCCAGGCCGGCCTGGATCAG & Construct $\operatorname{irp} 2 \Delta S 52$ deletion \\
\hline S3 & ATAAGATTGATGAGATGGTTAC & Construct $\operatorname{irp} 2 \Delta S 52$ deletion \\
\hline S4 & GCTCTAGATTCACGTAGCGTGGCGGGTTC & $\begin{array}{l}\text { Construct and check irp2 } 2 S 52 \\
\text { deletion }\end{array}$ \\
\hline YbtA-2 & GGGGTACCGACCTGGTTATCTCCCTG & Clone $y b t P$ promoter \\
\hline YbtP-2 & GGGGTACCGGGAGTAACTGAATTTCC & Clone $y b t P$ promoter \\
\hline YbtT For2 & GGCACTGCTGGCGAACGAG & Sequence $y b t T$ mutants \\
\hline YbtT Revl & GCGAGTCGTAGTGATAGC & Sequence $y b t T$ mutants \\
\hline YbtT Rev2 & CTTTCTGAAGTACTGGGCTG & Sequence $y b t T$ mutants \\
\hline
\end{tabular}

Y. pestis KIM6 + inv gene by allelic exchange and confirmed by hybridization to an $814 \mathrm{bp}$ EagI/EcoRI inv fragment isolated from pSucinv. The probe detected a 5242 bp BamHI fragment in wild-type strains and a $2578 \mathrm{bp}$ BamHI fragment in strains containing the integrated reporter. One reporter strain was selected and designated KIM6-2127+. An irp2:: kan2046 mutation on suicide plasmid pCIRP498.8 (Fetherston et al., 1995) was introduced into KIM62127 + by electroporation and allelic exchange. The resulting mutant did not synthesize Ybt and the strain was designated KIM6-2046.6.

PCR was used to delete the codon for serine 52 in the aryl carrier protein domain of HMWP2. Salicylate is attached to this residue via a phosphopantetheine cofactor (Gehring et al., 1998b; Keating et al., 2000). A 799 bp product upstream of the codon for $S 52$ was amplified using $P f u$ polymerase and primer pair S1 and S2 (Table 2). Primer pair S3 and S4 (Table 2) was used to amplify an 822 bp product downstream of the S52 codon. The products were purified using Zymo columns, treated with polynucleotide kinase and ligated together overnight at $15{ }^{\circ} \mathrm{C}$. The ligated products were used as a template for PCRs containing primers $\mathrm{S} 1$ and $\mathrm{S} 4$. The $\sim 1600 \mathrm{bp}$ fragment resulting from the amplification of the upstream product ligated to the downstream product was isolated from a low-melting agarose gel, digested with $B a m H I$ and $\mathrm{XbaI}$, and cloned into the same sites in pNEB193. A clone (pNEBirp2S) containing the correct insert with a deletion of S52 was confirmed by sequencing. The insert was transferred into the BamHI$X b a \mathrm{I}$ sites of pKNG101 to generate pKNGirp2 $\Delta$ S52.

The irp2 $\Delta S 52$ mutation was introduced into $Y$. pestis KIM6-2093 ( $\Delta y 2360::$ cam2093) by allelic exchange using pKNGirp2 $\Delta$ S52. Sucrose-resistant presumptive second cross isolates were replicaplated after overnight growth in $\mathrm{PMH} 2$ at $37{ }^{\circ} \mathrm{C}$ onto plates containing PMH2 or $\mathrm{PMH} 2$ with $50 \mu \mathrm{M}$ 2,2'-dipyridyl (DIP). Isolates which formed red colonies on $\mathrm{CR}$ agar and grew on $\mathrm{PMH}$ plates but not on PMH/DIP plates were screened by PCR using S4 and a primer which ends with the three deleted nucleotides $(\Delta \mathrm{irpS}$, Table 2). One of the strains which did not yield a PCR product was designated KIM6-2046.7. KIM6-2046.7 was grown in PMH2 for two transfers, along with KIM6 + and KIM6-2046.1 (irp2::kan2046.1). Filtered supernatants from these cultures were tested for the ability to stimulate the growth of KIM6-2046.1 incorporated into $\mathrm{PMH} 2$ plates containing $75 \mu \mathrm{M}$ DIP. KIM6 +, but not KIM6-2046.7, supernatants were able to promote the growth of KIM6-2046.1 on these plates, confirming the lack of Ybt synthesis by KIM6-2046.7.

Construction of alanine substitutions in YbtT. Four separate amino acid changes (G92A, G96A, S94A and H230A, using a predicted start of VTQS) were made in YbtT using pYbtT-H6 (Geoffroy et al., 2000; Table 1) with appropriate primers (Table 2) and a QuikChange II Site-Directed Mutagenesis kit (Stratagene) following the manufacturer's directions. DNA sequencing (Davis Sequencing) confirmed the alanine substitutions as well as the fidelity of the entire $y b t T$ ORF. Selected recombinant plasmids were designated pYbtT-G92A-H6, pYbtT-G96A-H6, pYbtT-S94A-H6 and pYbtT-H230A-H6 (Table 1). Western blot analysis was used to demonstrate expression levels of the YbtT substitution mutants.

Western blot analysis. For Western blot analysis, equal amounts of $Y$. pestis whole cells were suspended in sample buffer and boiled for $5 \mathrm{~min}$. Samples were separated by SDS-PAGE and immunoblotted to PVDF membranes. The blots were processed using a procedure modified from Towbin et al. (1979). Briefly, the membranes were blocked with $5 \%$ non-fat dry milk in $10 \mathrm{mM}$ Tris/ $\mathrm{HCl}(\mathrm{pH} \mathrm{7.6)}$, $137 \mathrm{mM} \mathrm{NaCl}$ and $0.1 \%$ Tween 20 (TBST), and then incubated with an appropriate antibody diluted in TBST with or without $0.1 \%$ gelatin. The blots were washed with TBST and incubated with horseradish peroxidase-conjugated protein A (Amersham Pharmacia Biotech). Immunoreactive proteins were detected with the ECL enhanced chemiluminescence Western blotting detection reagent (Amersham Pharmacia Biotech) and visualized on Kodak BioMax light film. Polyclonal rabbit sera against purified YbtE and the PCP1Cy2-PCP2 fragment of HMWP2 (Gehring et al., 1998b; Suo et al., 
1999) were generated by Animal Pharm Services. A YbtT peptide (CHAFSAMTALQKQPSTER) and corresponding polyclonal rabbit antisera against it were produced by Open Biosystems.

Bioassays for detection of Ybt synthesis. Three bioassays were used to detect the Ybt siderophore. (1) Culture supernatants were obtained from $Y$. pestis cells grown for six to nine generations in deferrated PMH2 at $37{ }^{\circ} \mathrm{C}$. Cells were pelleted by centrifugation and the supernatants filtered through a $0.2 \mu \mathrm{m}$ pore-size filter. Cells of $Y$. pestis KIM6-2046.1 (irp2: : kan2046.1), which are unable to synthesize the Ybt siderophore, were used to overlay PMH2-DIP (75 $\mu$ M DIP) plates solidified with $1 \%$ agarose as previously described, and cell-free culture supernatants $(25 \mu \mathrm{l})$ were added to wells in the plate. Growth responses to culture supernatants were monitored for $48-72 \mathrm{~h}$ (Bobrov et al., 2002; Fetherston et al., 1995).

(2) Cells of $Y$. pestis KIM6-2072 ( $\Delta y b t T 2072)$ carrying YbtT expression plasmids with and without alanine substitutions were assessed for their ability to promote the growth of KIM6-2046.1 at $37{ }^{\circ} \mathrm{C}$ by streaking the mutant strains adjacent to KIM6-2046.1 on solidified Brain Heart Infusion (BHI) containing 175-250 $\mu \mathrm{M}$ DIP (BHI-DIP). Prior to streaking, these $Y$. pestis strains were adapted to iron-deficient growth conditions as described above. KIM6-2046.1, which cannot produce the Ybt siderophore, is unable to grow on BHI-DIP at $37^{\circ} \mathrm{C}$ but can be cross-fed by Ybt-producing strains.

(3) Cells of $Y$. pestis KIM6-2072 ( $\Delta y b t T 2072)$ carrying YbtT expression plasmids with and without alanine substitutions were also assessed for their ability to grow under iron-chelated conditions using PMH2-DIP gradient plates $(0-100 \mu \mathrm{M}$ DIP $)$ at $37^{\circ} \mathrm{C}$. PMH2-DIP gradient plates were poured as previously described (Bearden \& Perry, 1999) using square $100 \mathrm{~mm}$ plates with six grids. Cells were acclimated to iron-deficient conditions as described above and spotted in each of the six grids across the DIP gradient, and growth at $37{ }^{\circ} \mathrm{C}$ was monitored daily for $48 \mathrm{~h}$.

Analysis of transcription of the ybtP: : lacZ reporter. Cells of $Y$. pestis KIM6-2046.6 (irp2::kan2046.1 inv::ybtP $P_{p}:$ lacZ) were grown overnight in deferrated $\mathrm{PMH} 2$ at $37{ }^{\circ} \mathrm{C}$, diluted to $\mathrm{OD}_{620} 0.1$ in fresh deferrated $\mathrm{PMH} 2$ and incubated at $37{ }^{\circ} \mathrm{C}$ to $\mathrm{OD}_{620} \sim 0.3$. At this point, cell-free spent supernatants from iron-deficient cultures of KIM6 + or selected $y b t$ biosynthetic mutants were added to a final volume of 10 or $50 \%(\mathrm{v} / \mathrm{v})$. Incubation at $37{ }^{\circ} \mathrm{C}$ was continued for $30 \mathrm{~min}$ before harvesting cells for $\beta$-galactosidase assays. $\beta$-Galactosidase activities in each culture were measured with a Genesys 5 spectrophotometer following cleavage of OPNG. Activities were expressed in Miller units (Miller, 1992). Each assay used two to three replicate samples, and the results are means from two or more independent cultures.

Ybt purification, standardization and determination. Ybt was extracted and purified from cell-free supernatants from iron-deficient cultures using a four-step modification of a procedure previously described (Miller et al., 2006). Supernatants and extracts were protected from light as much as possible while being handled. Approximately 1.51 of the supernatant was extracted with three $500 \mathrm{ml}$ washes of ethyl acetate. Ethyl acetate extracts were combined and the solvent was removed by rotary evaporation at $40{ }^{\circ} \mathrm{C}$. The resulting solid material was dissolved in a total of $15 \mathrm{ml} 100 \%$ ethanol, diluted to $50 \mathrm{ml}$ with water (distilled and de-ionized to $18 \mathrm{M} \Omega$ ) and then filtered through a $0.22 \mu \mathrm{m}$ pore-size filter. The filter was washed with $1-2 \mathrm{ml}$ ethanol.

The combined filtrate was purified with Sep-Pak C-18 mini preparative cartridges (Waters). Two cartridges for each set of samples were prepared by wetting with $5 \mathrm{ml}$ methanol followed by a $5 \mathrm{ml}$ water wash. The filtrate was loaded onto the cartridges in $25 \mathrm{ml}$ aliquots per cartridge, washed with $5 \mathrm{ml}$ water, and then eluted with two $5 \mathrm{ml}$ aliquots of methanol. Loading, washing and elution of the
C-18 cartridges were accomplished by gravity feed using a syringe body as a reservoir. The resulting methanol solution containing Ybt plus various impurities was reduced to $1 \mathrm{ml}$ total volume with a CentriVap vacuum concentrator (Labconco).

Ybt was then isolated using a Waters 600 HPLC equipped with a Waters 996 photodiode array UV/Vis detector and a $\mu \mathrm{RPC}$ C2/C18 ST 4.6/100 column (Amersham Biosciences). Initial injection conditions were $10 \mathrm{mM}$ ammonium formate, $\mathrm{pH} \mathrm{8.0,} \mathrm{for} \mathrm{all}$ concentrations of acetonitrile, immediately followed by $10 \mathrm{~min}$ with $10 \%$ acetonitrile, $160 \mathrm{~min}$ of a linear gradient from 10 to $25 \%$ acetonitrile, and ending with $5 \mathrm{~min}$ at $100 \%$ acetonitrile. The flow rate was $0.5 \mathrm{ml} \mathrm{min}^{-1}$. Spectrophotometric data were collected from 200 to $500 \mathrm{~nm}$. The baseline was monitored at $210 \mathrm{~nm}$. Fractions were collected at $1 \mathrm{~min}$ intervals in autoclaved $1.5 \mathrm{ml}$ microcentrifuge tubes and stored frozen at $-20{ }^{\circ} \mathrm{C}$ for later use. The column was reequilibrated between runs with $10 \mathrm{mM}$ ammonium formate, $\mathrm{pH}$ 8.0.

The collected $\mathrm{Fe}-\mathrm{Ybt}$-containing fractions were combined and loaded onto a C-18 reverse-phase cartridge prepared as described above. The $\mathrm{Fe}-\mathrm{Ybt}$ was eluted and the methanol removed by evaporation to dryness in a CentriVap vacuum concentrator. The resulting Ybt was diluted to $1.0 \mathrm{mg} \mathrm{ml}^{-1}$ in methanol. Serial dilutions of $0.05,0.025$ and $0.0125 \mathrm{mg} \mathrm{ml}^{-1}$ were prepared, and $50 \mu \mathrm{l}$ of each dilution plus a blank sample was injected into the HPLC system described above and monitored at $226 \mathrm{~nm}$. After elution, each peak area was integrated using Empower Pro software and a calibration curve generated by plotting peak area versus concentration with Microsoft Excel. Samples from each $Y$. pestis strain examined were extracted from $1.5 \mathrm{l}$ iron-deficient culture supernatant, purified and concentrated to $1 \mathrm{ml}$ as described above. A $100 \mu$ volume of concentrate from various Ybt biosynthetic mutants was prepared as described above, and the resulting peaks were integrated and compared with the authentic Ybt calibration curve.

\section{RESULTS}

\section{Transcriptional activation of the $y b t P:$ : lacZ reporter}

While most $y b t$ biosynthetic mutants exhibit reduced transcription of a plasmid-encoded $y b t P::$ lac $Z$ reporter, we previously reported that three biosynthetic mutations [ $\Delta y b t T, y b t S:$ : kan and irp1-2086 irp1-TE)] still result in the higher levels of $y b t P$ transcription normally associated with Ybt siderophore synthesis. However, these three mutants are phenotypically similar to other biosynthetic mutants in that they fail to grow at $37{ }^{\circ} \mathrm{C}$ under iron-chelated conditions and culture supernatants do not stimulate growth of a siderophore-negative indicator strain (KIM62046.1) of $Y$. pestis in our Ybt bioassay. We previously proposed that the two TE mutants might make lower levels of authentic Ybt and that the ybtS::kan mutant may produce an aberrant Ybt-like molecule with another aromatic group replacing salicylate (Bobrov et al., 2002; Geoffroy et al., 2000; Perry et al., 2003a). Since transcriptional activation of the $y b t P$ promoter is $\sim 500$-fold more sensitive than growth stimulation in our Ybt bioassay (Fetherston et al., 1996; Geoffroy et al., 2000; Perry et al., 2003a), we decided to use transcriptional activity of the $y b t P$ promoter to test culture supernatants for the presence of authentic Ybt or a Ybt-like molecule capable of acting as a signal molecule but not as a growth factor for ironstarved $Y$. pestis cells. 
We constructed KIM6-2046.6, which has an irp2::kan mutation so that it cannot synthesize $\mathrm{Ybt}$ and a chromosomally integrated $y b t P:$ : lac $Z$ reporter. This strain was exposed for $30 \mathrm{~min}$ to culture supernatants at a final concentration of $10 \%(\mathrm{v} / \mathrm{v})$. The $\beta$-galactosidase activity of cells exposed to the $\Delta y b t T$ culture supernatant was equivalent to those exposed to KIM6+, which produces normal levels of Ybt (Fig. 2). However, culture supernatants from the $y b t S$ and irp2-TE mutants caused a less than twofold activation of the transcriptional reporter compared with the KIM6 ( $\Delta p g m)$ negative control (Fig. 2). When we increased the concentration of our spent supernatants to $50 \%(\mathrm{v} / \mathrm{v})$, the $y b t P:$ : lac $Z$ reporter activity was significantly increased by both the $y b t S:$ : kan and irp2-TE mutant culture supernatants (Fig. 2). These results indicate that all three mutants still synthesize and secrete $\mathrm{Ybt}$ or a Ybt-like molecule that acts in signal transduction, but that the amount of this compound(s) (or its efficacy) in the culture supernatants varies among the different mutants.

\section{Purification of Ybt}

A modified Ybt purification scheme (Methods; Miller et al., 2006) was used to determine whether $y b t S, y b t T$ and irp1TE mutants produce low levels of authentic Ybt. The elution profile of Ybt from KIM6 + is shown in Fig. 3(a).

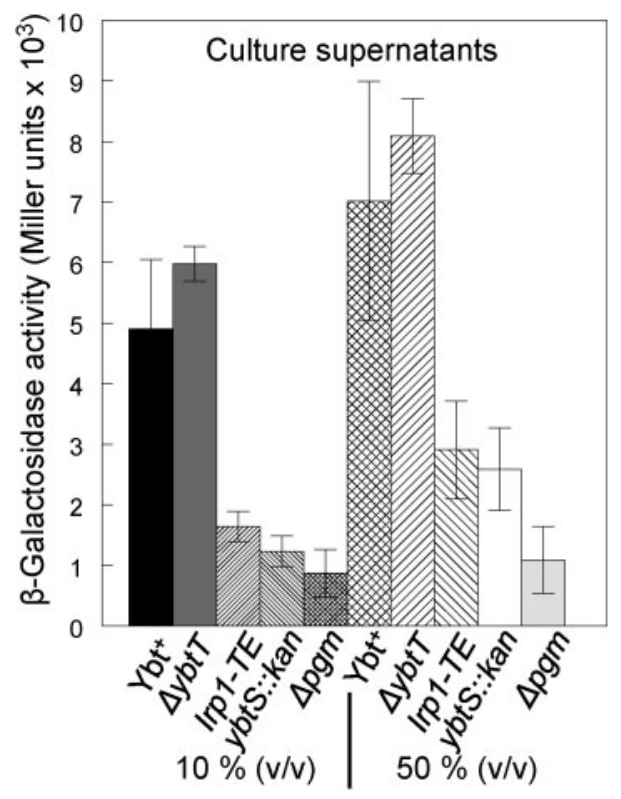

Fig. 2. $\beta$-Galactosidase activities from a $y b t P$ : : lac $Z$ reporter in $Y$. pestis strains. Activities of a chromosomally integrated $y b t P$ : : lac $Z$ reporter in KIM6-2046.6 after 30 min of exposure to spent culture supernatants ( 10 and $50 \%, v / v)$ from the indicated strains. Reporter strain KIM6-2046.6 has an irp2 : : kan2046.1 mutation and does not produce Ybt. Strains: $\mathrm{Ybt}^{+}, \mathrm{KIM} 6+; \Delta y b t T$, KIM6-2072; irp1-TE, KIM6-2086; ybtS : : kan, KIM6-2070.1; $\Delta$ pgm, KIM6.
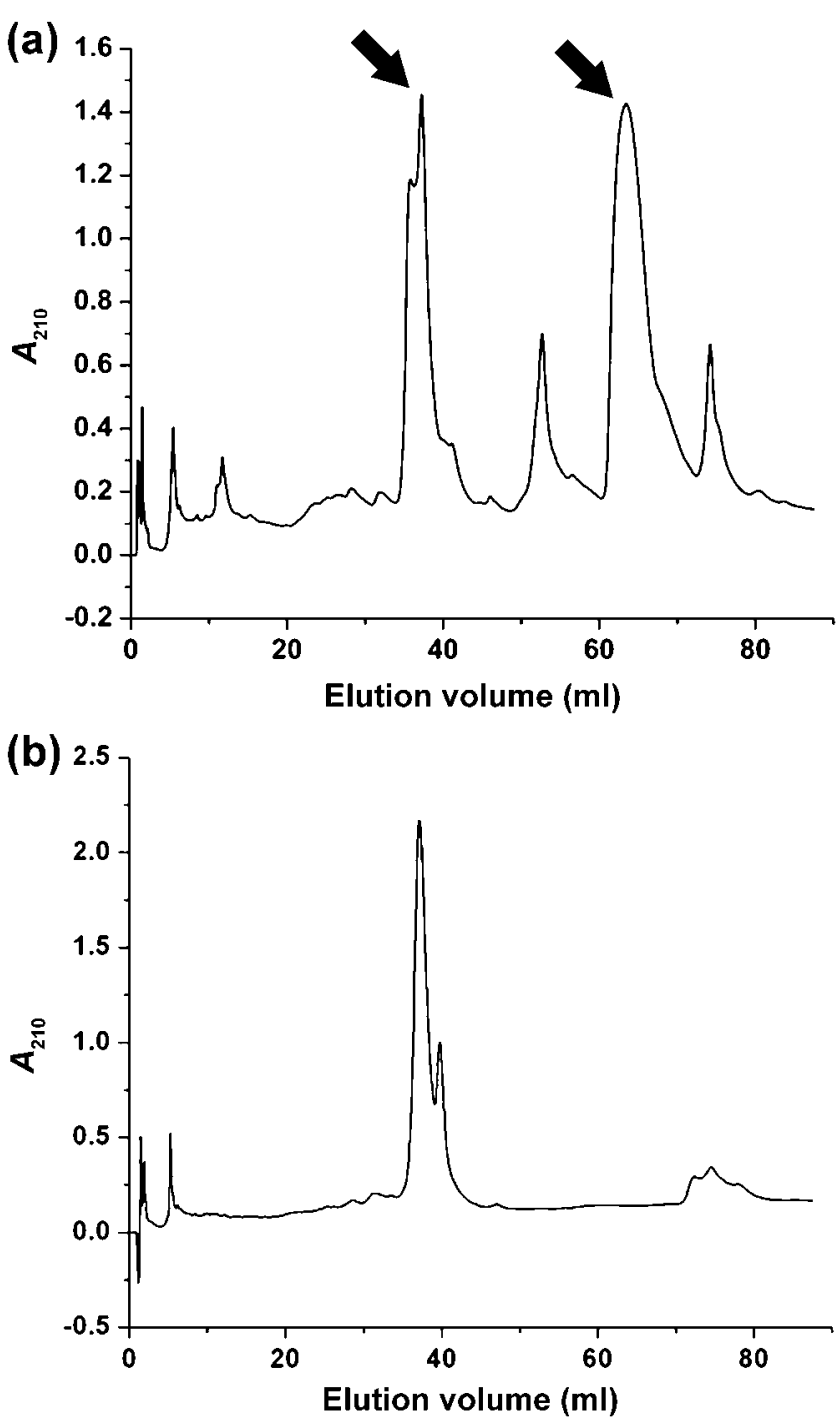

Fig. 3. HPLC analysis of KIM6 + supernatant without (a) and with (b) added iron. Addition of iron approximately doubles the size of the peak at $37.5-40 \mathrm{ml}$ (b) and eliminates the peak at $65 \mathrm{ml}$ (a). Arrows in (a) show the apo $(65 \mathrm{ml})$ and ferrated $(37.5-40 \mathrm{ml})$ forms of Ybt.

Elution with a 0-100\% acetonitrile gradient yielded multiple peaks (Fig. 3a). An iron-rich extract was prepared by mixing $100 \mu \mathrm{l} 0.10 \mathrm{M} \mathrm{FeCl}_{3}$ with an equal volume of the KIM6 + extract and $800 \mu$ methanol. The chromatogram of this sample eliminated all but the double peak at 37.5 and $40 \mathrm{ml}$, which doubled in area (Fig. 3b). The identity of the Ybt molecules was confirmed by directly extracting the UV/visible spectrum from the data provided by the Waters 996 detector using Waters Empower Pro software and by positive ion electrospray ionization (ESI)-MS (University of Kentucky Mass Spectrometry Facility). These analyses indicated that ferric-Ybt and deferrated Ybt eluted at $\sim 37.5-40 \mathrm{ml}$ and $65 \mathrm{ml}$, respectively (arrows in Fig. 3a), with the expected UV/visible spectra (Fig. 4). 

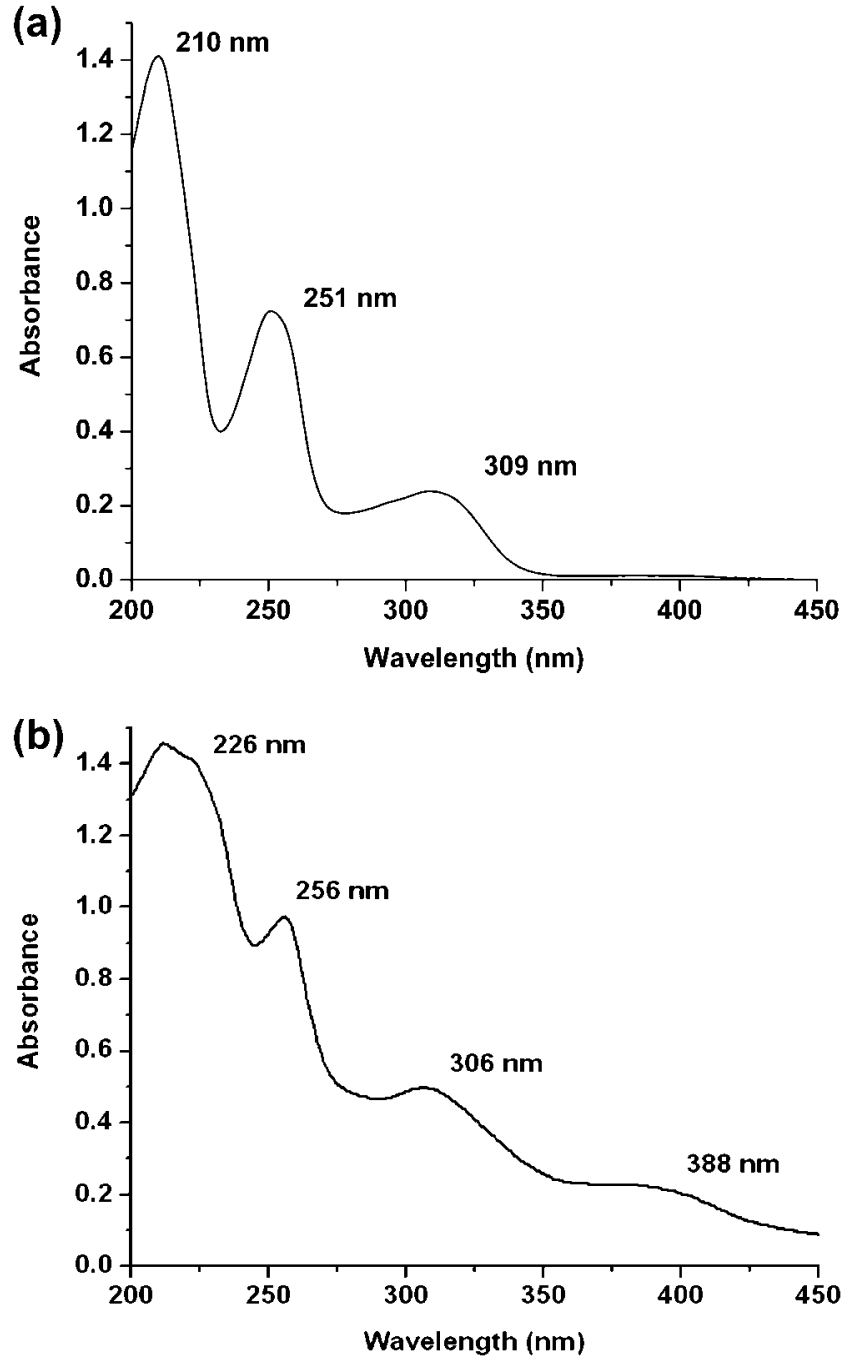

Fig. 4. UV/visible spectra from the peaks at approximately $65 \mathrm{ml}$ (a) and 37.5-40 $\mathrm{ml}$ (b) in Fig. 3(a). Based on the data presented by Chambers et al. (1996), the peak at $65 \mathrm{ml}$ is the apo form of Ybt while the peak at $37.5-40 \mathrm{ml}$ is ferrated Ybt. The identifying characteristics of the apo form are the presence of three distinct maxima at 210, 251 and $309 \mathrm{~nm}$ (a), while the ferrated form has four distinct maxima at 226, 256, 306 and $388 \mathrm{~nm}$ (b).

KIM6 $+\left(\mathrm{Ybt}^{+}\right)$yielded $12.9 \mu \mathrm{g} \mathrm{Ybt}^{-1}$, while KIM6-2072 ( $\Delta y b t T 2072)$ gave a reduced yield of $3.0 \mu \mathrm{g} \mathrm{l}^{-1}$. In contrast, the irp1-TE mutant (lacking the TE activity proposed to release the completed Ybt molecule) had a greatly reduced yield of $0.37 \mu \mathrm{g} \mathrm{l}^{-1}$. Both KIM6 ( $\left.\Delta p g m\right)$ and KIM6-2070.1 $(y b t S::$ kan2070) had an undetectable level of Ybt with a detection limit of $0.01 \mu \mathrm{g} \mathrm{l} \mathrm{l}^{-1}$. The amount of Ybt secreted by the two TE mutants corresponds to the level of transcriptional activity observed with crude supernatants (see above). The residual transcriptional activity of the $y b t S:$ : kan mutant (see $50 \%$ supernatant in Fig. 2) could be due to the production of an aberrant Ybt-like molecule with signalling activity.

\section{YbtT and Ybt synthesis}

The YbtT predicted type II TE is thought to be involved in editing and removal of incorrect precursor molecules from HMWP1 and HMWP2. YbtT would therefore contribute to the efficiency of Ybt synthesis by relieving biosynthetic bottlenecks through removal of incorrect precursor molecules from the Ybt synthetase complex. The decrease in Ybt production in a $y b t T$ mutant indicates that this enzyme is required for optimal Ybt synthesis (see above). To more directly link reduced Ybt synthesis in the $y b t T$ mutant with the predicted TE activity of $\mathrm{YbtT}$, we mutated several residues associated with type II TE activity in other enzymes. While predicted type II TE domains span several hundred residues, alignments identified 12 invariant and 10 highly conserved $(>80 \%)$ residues (Kotowska et al., 2009; Linne et al., 2004). These include a nucleophile elbow motif (G-x$\mathrm{Nu}-\mathrm{x}-\mathrm{G}$; GxSxG residues 92-96 in YbtT) and a histidine residue at position $230 \mathrm{in} \mathrm{YbtT.} \mathrm{The} \mathrm{actual} \mathrm{importance} \mathrm{of}$ these residues to YbtT function has never been assessed. Consequently, we constructed separate alanine substitution mutations in each of these four residues in our YbtTexpressing plasmid pYbtT-H6, which complements the inframe $y b t T$ mutant strain KIM6-2072 (Geoffroy et al., 2000).

Cross-feeding studies indicated that the YbtT-H230A and YbtT-S94A variant proteins were little better than the negative control in providing Ybt siderophore for growth of a $Y$. pestis Ybt biosynthetic (irp2) mutant. The two individual glycine substitutions (YbtT-G92A and YbtTG96A) provided more growth stimulation, but not as much as the YbtT-H6 positive control (data not shown). To better quantify the ability of each substitution mutant to produce Ybt, we examined the growth of KIM6 + and the $\mathrm{YbtT}^{-}$ mutant bearing various YbtT-H6 plasmids across a 0 $100 \mu \mathrm{M}$ DIP gradient plate (Fig. 5). Expression of YbtT-H6 allowed growth further into the DIP gradient than the $\mathrm{Ybt}^{+}$ strain KIM6 +, possibly due to increased expression from the pPROEX-1 vector. The histidine and serine substitution mutants, which had YbtT protein levels similar to those of the mutant expressing YbtT-H6 from pPROEX-1 (data not shown), showed growth similar to that of the in-frame $y b t T$ mutant, which was significantly reduced compared with the YbtT-H6-expressing strain (Fig. 5). In contrast, KIM6+ and the two strains expressing the glycine-substituted $\mathrm{YbtT}$ variants had similar levels of YbtT protein expression (data not shown) and grew into similar DIP concentrations in the gradient (Fig. 5). These results correspond to the crossfeeding analysis and suggest that G92 and G96 are not essential for YbtT activity while $\mathrm{H} 230$ and S94 have a significant effect on Ybt synthesis.

\section{Aberrant Ybt-like molecules}

YbtS is responsible for synthesizing salicylate, the molecule that presumably initiates Ybt synthesis. A strain with a mutation in $y b t S$ does not produce any detectable authentic Ybt, yet $y b t$ reporters in this mutant are fully activated (Fig. 6; Geoffroy et al., 2000; Perry et al., 2003a), a 


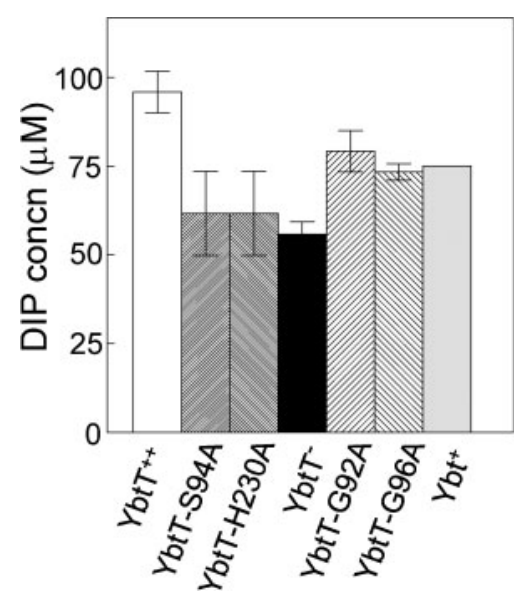

Fig. 5. Growth of $Y$. pestis strains at $37{ }^{\circ} \mathrm{C}$ across PMH2-DIP gradient plates $(0-100 \mu \mathrm{M}$ DIP). The results represent the mean from two independent experiments of incremental growth across the gradient by $48 \mathrm{~h}$ of incubation. Error bars, SD. Strains: $\mathrm{YbtT}^{++}$, KIM6-2072(pYbtT-H6) positive control; YbtT-S94A, KIM6-2072(pYbtT-S94A-H6); YbtT-H230A, KIM6-2072(pYbtTH230A-H6); YbtT $^{-}$, KIM6-2072 ( $\left.\Delta y b t T 2072\right)$ negative control; YbtT-G92A, KIM6-2072(pYbtT-G92A-H6); YbtT-G96A, KIM62072(pYbtT-G96A-H6); $\mathrm{Ybt}^{+}, \mathrm{KIM} 6^{+}$positive control.

process which responds to Ybt. It is possible that another enzyme synthesizes a phenolate compound that ineffectively substitutes for salicylate. Encoded within the $Y$. pestis KIM pgm locus, Y2336 shows a high degree of similarity to YbtS: both proteins have anthranilate synthase domains (Deng et al., 2002). However, a ybtS:: kan $\Delta y 2336$ double mutant (KIM6-2070.2) still fully activated transcription from a $y b t P::$ lacZ reporter (Fig. 6). Consequently, if an alternative aromatic component is incorporated by the $y b t S:$ : kan mutant it is not dependent upon the $y 2336$ gene product for this activity.

To test whether a Ybt-like molecule possessing only the thiazolidine and thiazoline rings without a substitute salicylate component might be capable of transcriptional activation, we deleted serine residue 52 of HMWP2 (strain KIM6-2046.7), which would eliminate tethering of salicylate or a salicylate-like component for incorporation into authentic Ybt or a Ybt-like molecule. In contrast to the $y b t S:$ :kan $\Delta y 2336$ double mutant, KIM6-2046.7 showed reduced transcription from a $y b t P$ reporter (data not shown) and little or no YbtE or HMWP2 proteins by Western blot analysis, in the absence of added Ybt (Fig. 7). Synthesis of these two proteins in KIM6-2046.7 and the inframe irp2 mutant, but not in the polar irp2:: kan mutant, was restored following a $2 \mathrm{~h}$ exposure to purified Ybt (Fig. 7). The higher levels of HMWP2 and $\mathrm{YbtE}$ in $\mathrm{Ybt}^{+}$ $\mathrm{KIM} 6+$ and the $y b t S:: k a n$ mutant are likely due to the continuous presence of endogenous Ybt (or Ybt-like) molecules. These results suggest that the irp2 $\Delta S 52$ mutant does not make a compound capable of stimulating the transcription of $y b t$ promoters.

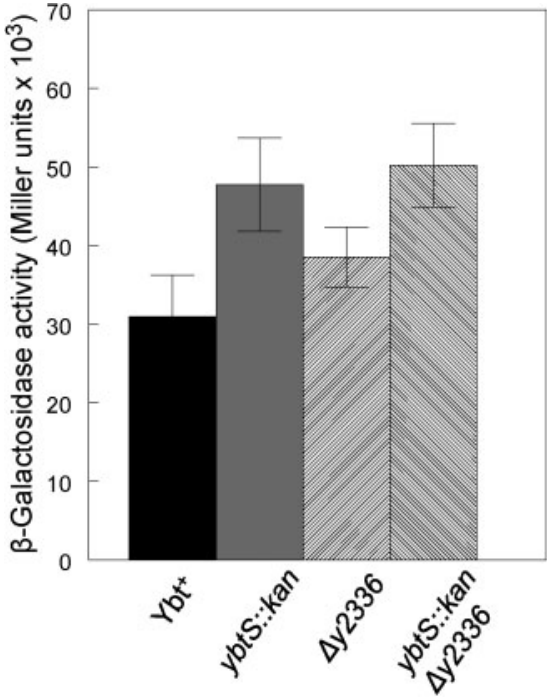

Fig. 6. $\beta$-Galactosidase activities from a $y b t P:$ : lac $Z$ reporter in $Y$. pestis strains. Activities of a plasmid-encoded ybtP: : lac $Z$ reporter $(\mathrm{pEUYbtP})$ in the control $\left(\mathrm{Ybt}^{+}\right)$and indicated mutant strains. Endogenous synthesis of Ybt or a Ybt-like molecule is responsible for transcriptional activation. Strains: $\mathrm{Ybt}^{+}, \mathrm{KIM} 6+; y b t S:$ : kan, KIM6-2070.1; $\Delta y 2336$, KIM6-2126; ybtS: : kan $\Delta y 2336$, KIM62070.2 .

Finally, we tested whether the signalling molecule present in the supernatant from the $y b t S:: k a n$ mutant was extracted during our purification procedure. Ethyl acetate extracts from equal volumes of culture supernatants of the $y b t S: k a n, y b t T$ and $\operatorname{irp1-TE}$ mutants as well as KIM6+ $\left(\mathrm{Ybt}^{+}\right)$and KIM6 ( $\left.\Delta p g m, \mathrm{Ybt}^{-}\right)$were concentrated 1000fold. Serial 10-fold dilutions of the concentrated extracts were added to the reporter strain KIM6-2046.6. Addition

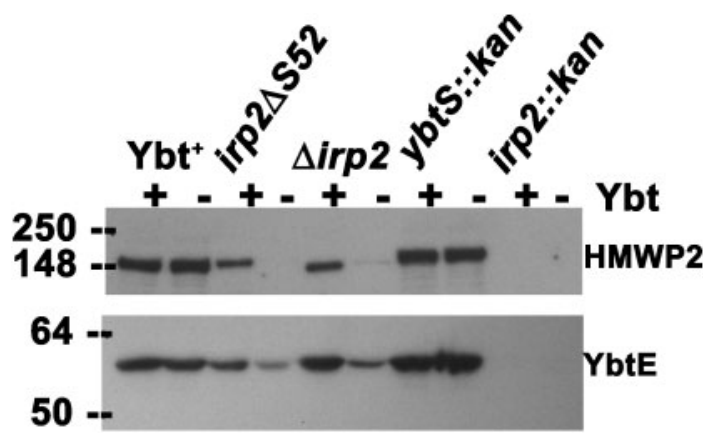

Fig. 7. Western blot analysis of HMWP2 and YbtE expression in $Y$. pestis strains in the presence and absence of exogenous $Y b t$. Equal concentrations of whole-cell lysates were separated by SDS-PAGE; immunoblots were reacted with antiserum against HMWP2 or YbtE, as indicated. Strains: $\mathrm{Ybt}^{+}, \mathrm{KIM6}+; \Delta$ irp2 (inframe), KIM6-2046.3; irp2 $\Delta$ S52, KIM6-2046.7; ybtS : : kan, KIM62070.1; irp2: : kan (polar), KIM6-2046.1. 
of $0.01 \mu \mathrm{l}$ of the extract from the Ybt-producing strain $\mathrm{KIM} 6+$ caused maximal activation of the ybtP:: lacZ reporter. Extracts from the $y b t T$ and irp1-TE mutants required 10- and 100-fold higher volumes for maximal activation, respectively. These results approximately correspond to the amount of authentic Ybt produced by these mutants (see above). A 100-fold higher volume of the $\Delta y b t S$ extract also was required for maximal activation of the reporter (data not shown).

Surprisingly, the concentrated extract from the $\mathrm{Ybt}^{-}$strain KIM6 also stimulated transcription from the reporter but required a 1000-fold higher concentration than the extract from the Ybt-producing strain to do so. Extracted and concentrated uninoculated $\mathrm{PMH} 2$ provided no activation of the $y b t P$ : : lac $Z$ reporter (data not shown). These results indicate that KIM6, which lacks all the Ybt biosynthetic genes except for $y b t D$, produces a non-Ybt molecule capable of signalling activity.

\section{DISCUSSION}

In the presence of $\mathrm{Ybt}, \mathrm{YbtA}$ activates transcription of transport and biosynthetic genes while repressing transcription from its own promoter. The expression of several siderophore-dependent iron-transport systems is controlled by the interaction of the cognate siderophore with its outer membrane receptor, which transmits a TonBdependent signal that activates a regulator for that system (Braun, 2001; Crosa, 1997; Venturi et al., 1995). However, in $Y$. pestis, three separate $\Delta p s n$ mutations have no effect on YbtA-regulated gene expression. In addition, signal transduction is not affected by a $Y$. pestis ton $B$ mutation (Bearden et al., 1997; Perry et al., 2003a, b). Thus, it is unlikely that binding of Ybt to the outer membrane receptor transmits a signal that then activates YbtA.

Since YbtA is a member of the AraC family of transcriptional regulators, we have proposed that YbtA binds the siderophore and that this complex directly affects the transcription of the regulated genes of the Ybt system (Fig. 1b). During the Ybt purification process, much of the siderophore is saturated with iron and more of the apo form would be converted to ferri-Ybt after addition of the purified siderophore to $\mathrm{PMH} 2$, which contains $\sim 0.3 \mu \mathrm{M}$ iron. Consequently, we have proposed that it is the ironchelated form of Ybt that serves as the signalling molecule. This model corresponds to the pyochelin- and PchRdependent regulation demonstrated for that system in Pseudomonas aeruginosa (Michel et al., 2005). The Bordetella alcaligin system also requires an AraC-type regulator, AlcR, and the alcaligin siderophore for transcriptional activation of the system. In contrast to the other two systems, it appears that the iron-free form of alcaligin is the signalling molecule (Brickman \& Armstrong, 2002, 2009; Brickman et al., 2001).

In an apparent contradiction to our model, $y b t S, y b t T$ and irp1-TE mutations cause a loss of Ybt synthesis without affecting YbtA regulation. We had proposed that these three mutants produce either low levels of Ybt that are sufficient for activation or aberrant Ybt-like molecules that are not detectable by the bioassay but can bind to YbtA and activate transcription (Bearden et al., 1997; Fetherston et al., 1996; Geoffroy et al., 2000; Perry et al., 2001, 2003a, b). Our results in this study indicate that the $y b t T$ and irp1-TE mutants do produce sufficient Ybt to activate transcription.

YbtT is a putative type II TE that likely provides an editing function to remove aberrant molecules from the enzyme complex. In this study we have demonstrated that a $y b t T$ mutant synthesizes authentic Ybt but at levels $\sim 25 \%$ of that of the parent strain. Mutations in other type II TEs cause similar reductions in the products of NRPSs and PKSs (Butler et al., 1999; Leduc et al., 2007; Schneider \& Marahiel, 1998). Our results suggest that Ybt synthesis is relatively error-prone; this conclusion is supported by an apparent increase in Ybt levels due to increased expression of YbtT (Fig. 5). Miller et al. (2002) synthesized Ybt in vitro using appropriate precursors and four enzymes (YbtE, HMWP1, HMWP2 and YbtU). Including YbtT in the in vitro reaction did not increase the biosynthetic rate. However, the in vitro reaction only contained the appropriate substrates for Ybt synthesis. Perhaps in vivo, improper substrates are added to the HMWP1 and HMWP2 scaffold, in which case YbtT would be required for their removal.

A TE catalytic triad of Ser-Asp-His has been identified in type II TEs such as Bacillus subtilis SrfA and Streptomyces coelicolor ScoT. Alignments indicate that $\mathrm{YbtT}$ also possesses a putative catalytic triad: S94-D172-H230 (Kotowska et al., 2009; Linne et al., 2004). Alanine substitutions at S94 and $\mathrm{H} 230$ caused an iron-chelated growth defect similar to that of the $y b t T$ mutant (Fig. 5). In addition to S94, the nucleophile elbow of YbtT ( $\mathrm{GxSxG}$ ) contains two invariant glycines. However, substitution of an alanine for either of these two residues did not significantly affect the ability of $Y$. pestis to grow under iron-chelating conditions. While these residues are likely not critical to the TE activity of YbtT, they did have an effect on YbtT protein levels. Although expressed from a moderate-copy-number plasmid, Western blot analysis indicated the two substituted proteins had levels similar to that of YbtT expressed from the single chromosomal gene (data not shown).

The irp1-TE mutant also synthesizes and secretes authentic Ybt but at levels only $\sim 3 \%$ of that of the parent strain. In this mutant most of the completed Ybt molecule likely remains tethered to HWMP1. Either sufficient quantities are released to stimulate transcription or Ybt tethered to the enzyme complex can serve as a signalling molecule.

In contrast, the $y b t S$ mutant does not make detectable levels of authentic Ybt but still produces a signalling molecule. The activity of a Ybt-activated reporter in the $y b t S$ mutant is equivalent to that of the parental strain, although ethyl acetate extracts of culture supernatants from 
this mutant require 100-fold higher volumes for maximal activation of transcription from the $y b t P$ promoter. This suggests that the aberrant Ybt-like molecule is not secreted effectively and/or not efficiently extracted into ethyl acetate. The inability of an irp2 $\Delta S 52$ mutant to activate transcription of Ybt-regulated genes indicates that a Ybtlike molecule simply lacking salicylate is not made as the signalling molecule. This result is in agreement with in vitro studies that demonstrate that reactions containing purified HMWP2 with a S52A substitution do not yield a product (Keating et al., 2000). In the ybtS mutant, a different phenolate compound is most likely substituted for salicylate. We have demonstrated that the $y b t S$-like gene, $y 2336$, does not encode a product that is responsible for the synthesis of this compound.

Surprisingly, a $\Delta p g m$ mutant (which lacks all $y b t$ genes except for $y b t D$ ) also produced a compound capable of activating transcription. The following reasons suggest that this compound is different from the Ybt-like signalling molecule produced by the $y b t S$ mutant. First, the $y b t S$ mutant produces sufficient levels of the compound to fully activate transcription of the $y b t P:: l a c Z$ reporter carried in that strain. Only background levels of activity are detected in a $\Delta p g m$ mutant. However, $\Delta p g m$ strains lack the transcriptional activator YbtA in addition to Ybt. Another biosynthetic mutant, such as $\operatorname{irp} 2:: \mathrm{kan}$, is a fairer comparison with the $y b t S$ mutant. Neither of these mutants makes Ybt, but they still encode YbtA; yet the activity of a $y b t P:: l a c Z$ reporter in the $y b t S$ mutant is about 20 -fold higher than in an irp2::kan mutant. Indeed, if the $\Delta p g m$ compound were the same as that present in the $y b t S$ mutant then all biosynthetic mutants would be expected to show the same level of endogenous transcriptional activation, yet they do not. Second, in concentrated ethyl acetate extracts from the two mutants, the $y b t S$ mutant compound is $\sim 10$-fold more effective or abundant than the $\Delta p g m$ compound. Note that the $y b t S$ mutant probably produces both Ybt-like compounds. Regardless, isolation of these compounds will be required to definitively demonstrate that there are two separate non-Ybt compounds capable of Ybt signalling. While these compounds are not biologically relevant to normal Ybt regulation, a comparison of the structures of Ybt and other signalling molecules will help in determining the chemical structures recognized as a Ybt signal.

\section{ACKNOWLEDGEMENTS}

This study was supported by Public Health Service grant AI033481 from the National Institutes of Health. We thank the Chris Walsh research group (Harvard Medical School) for generously providing purified YbtE protein and the purified PCP1-Cy2-PCP2 fragment of HMWP2.

\section{REFERENCES}

Anisimov, R., Brem, D., Heesemann, J. \& Rakin, A. (2005). Molecular mechanism of YbtA-mediated transcriptional regulation of divergent overlapping promoters $y b t A$ and irp6 of Yersinia enterocolitica. FEMS Microbiol Lett 250, 27-32.

Bearden, S. W. \& Perry, R. D. (1999). The Yfe system of Yersinia pestis transports iron and manganese and is required for full virulence of plague. Mol Microbiol 32, 403-414.

Bearden, S. W., Fetherston, J. D. \& Perry, R. D. (1997). Genetic organization of the yersiniabactin biosynthetic region and construction of avirulent mutants in Yersinia pestis. Infect Immun 65, 16591668.

Beesley, E. D., Brubaker, R. R., Janssen, W. A. \& Surgalla, M. J. (1967). Pesticins. III. Expression of coagulase and mechanism of fibrinolysis. J Bacteriol 94, 19-26.

Bobrov, A. G., Geoffroy, V. A. \& Perry, R. D. (2002). Yersiniabactin production requires the thioesterase domain of HMWP2 and $\mathrm{YbtD}$, a putative phosphopantetheinylate transferase. Infect Immun 70, 42044214.

Braun, V. (2001). Iron uptake mechanisms and their regulation in pathogenic bacteria. Int J Med Microbiol 291, 67-79.

Braun, V. (2005). Bacterial iron transport related to virulence. Contrib Microbiol 12, 210-233.

Brickman, T. J. \& Armstrong, S. K. (2002). Bordetella interspecies allelic variation in AlcR inducer requirements: identification of a critical determinant of AlcR inducer responsiveness and construction of an alcR(Con) mutant allele. J Bacteriol 184, 1530-1539.

Brickman, T. J. \& Armstrong, S. K. (2009). Temporal signaling and differential expression of Bordetella iron transport systems: the role of ferrimones and positive regulators. Biometals 22, 33-41.

Brickman, T. J., Kang, H. Y. \& Armstrong, S. K. (2001). Transcriptional activation of Bordetella alcaligin siderophore genes requires the AlcR regulator with alcaligin as inducer. J Bacteriol 183, 483-489.

Bullen, J. J., Rogers, H. J., Spalding, P. B. \& Ward, C. G. (2005). Iron and infection: the heart of the matter. FEMS Immunol Med Microbiol 43, 325-330.

Butler, A. R., Bate, N. \& Cundliffe, E. (1999). Impact of thioesterase activity on tylosin biosynthesis in Streptomyces fradiae. Chem Biol 6, 287-292.

Chambers, C. E., McIntyre, D. D., Mouck, M. \& Sokol, P. A. (1996). Physical and structural characterization of yersiniophore, a siderophore produced by clinical isolates of Yersinia enterocolitica. Biometals 9, 157-167.

Crosa, J. H. (1997). Signal transduction and transcriptional and posttranscriptional control of iron-regulated genes in bacteria. Microbiol Mol Biol Rev 61, 319-336.

Crosa, J. H. \& Walsh, C. T. (2002). Genetics and assembly line enzymology of siderophore biosynthesis in bacteria. Microbiol Mol Biol Rev 66, 223-249.

Deng, W., Burland, V., Plunkett, G., III, Boutin, A., Mayhew, G. F., Liss, P., Perna, N. T., Rose, D. J., Mau, B. \& other authors (2002). Genome sequence of Yersinia pestis KIM. J Bacteriol 184, 4601-4611.

Drechsel, H., Stephan, H., Lotz, R., Haag, H., Zähner, H., Hantke, K. \& Jung, G. (1995). Structure elucidation of yersiniabactin, a siderophore from highly virulent Yersinia strains. Liebigs Ann 1995, 1727-1733.

Fetherston, J. D., Schuetze, P. \& Perry, R. D. (1992). Loss of the pigmentation phenotype in Yersinia pestis is due to the spontaneous deletion of $102 \mathrm{~kb}$ of chromosomal DNA which is flanked by a repetitive element. Mol Microbiol 6, 2693-2704.

Fetherston, J. D., Lillard, J. W., Jr \& Perry, R. D. (1995). Analysis of the pesticin receptor from Yersinia pestis: role in iron-deficient growth and possible regulation by its siderophore. J Bacteriol 177, $1824-1833$. 
Fetherston, J. D., Bearden, S. W. \& Perry, R. D. (1996). YbtA, an AraC-type regulator of the Yersinia pestis pesticin/yersiniabactin receptor. Mol Microbiol 22, 315-325.

Fetherston, J. D., Bertolino, V. J. \& Perry, R. D. (1999). YbtP and YbtQ: two $\mathrm{ABC}$ transporters required for iron uptake in Yersinia pestis. Mol Microbiol 32, 289-299.

Fetherston, J. D., Kirillina, O., Bobrov, A. G., Paulley, J. T. \& Perry, R. D. (2010). The yersiniabactin transport system is critical for the pathogenesis of bubonic and pneumonic plague. Infect Immun 78, 2045-2052.

Gehring, A. M., DeMoll, E., Fetherston, J. D., Mori, I., Mayhew, G. F., Blattner, F. R., Walsh, C. T. \& Perry, R. D. (1998a). Iron acquisition in plague: modular logic in enzymatic biogenesis of yersiniabactin by Yersinia pestis. Chem Biol 5, 573-586.

Gehring, A. M., Mori, I., Perry, R. D. \& Walsh, C. T. (1998b). The nonribosomal peptide synthetase HMWP2 forms a thiazoline ring during biogenesis of yersiniabactin, an iron-chelating virulence factor of Yersinia pestis. Biochemistry 37, 11637-11650.

Geoffroy, V. A., Fetherston, J. D. \& Perry, R. D. (2000). Yersinia pestis $\mathrm{YbtU}$ and $\mathrm{YbtT}$ are involved in synthesis of the siderophore yersiniabactin but have different effects on regulation. Infect Immun 68, 4452-4461.

Gong, S., Bearden, S. W., Geoffroy, V. A., Fetherston, J. D. \& Perry, R. D. (2001). Characterization of the Yersinia pestis Yfu ABC iron transport system. Infect Immun 69, 2829-2837.

Kaniga, K., Delor, I. \& Cornelis, G. R. (1991). A wide-host-range suicide vector for improving reverse genetics in Gram-negative bacteria: inactivation of the blaA gene of Yersinia enterocolitica. Gene 109, 137-141.

Keating, T. A., Miller, D. A. \& Walsh, C. T. (2000). Expression, purification, and characterization of HMWP2, a $229 \mathrm{kDa}$, six domain protein subunit of yersiniabactin synthetase. Biochemistry 39, 47294739

Kerbarh, O., Ciulli, A., Howard, N. I. \& Abell, C. (2005). Salicylate biosynthesis: overexpression, purification, and characterization of Irp9, a bifunctional salicylate synthase from Yersinia enterocolitica. J Bacteriol 187, 5061-5066.

Kotowska, M., Pawlik, K., Smulczyk-Krawczyszyn, A., BartoszBechowski, H. \& Kuczek, K. (2009). Type II thioesterase ScoT, associated with Streptomyces coelicolor A3(2) modular polyketide synthase Cpk, hydrolyzes acyl residues and has a preference for propionate. Appl Environ Microbiol 75, 887-896.

Leduc, D., Battesti, A. \& Bouveret, E. (2007). The hotdog thioesterase EntH (YbdB) plays a role in vivo in optimal enterobactin biosynthesis by interacting with the ArCP domain of EntB. J Bacteriol 189, 71127126.

Lesic, B. \& Carniel, E. (2004). The high pathogenicity island: a broadhost-range pathogenicity island. In Yersinia Molecular and Cellular Biology, pp. 285-306. Edited by E. Carniel \& B. J. Hinnebusch. Wymondham, UK: Horizon Bioscience.

Linne, U., Schwarzer, D., Schroeder, G. N. \& Marahiel, M. A. (2004). Mutational analysis of a type II thioesterase associated with nonribosomal peptide synthesis. Eur J Biochem 271, 1536-1545.

Michel, L., González, N., Jagdeep, S., Nguyen-Ngoc, T. \& Reimmann, C. (2005). PchR-box recognition by the AraC-type regulator PchR of Pseudomonas aeruginosa requires the siderophore pyochelin as an effector. Mol Microbiol 58, 495-509.

Miethke, M. \& Marahiel, M. A. (2007). Siderophore-based iron acquisition and pathogen control. Microbiol Mol Biol Rev 71, 413-451.

Miller, J. H. (1992). A Short Course in Bacterial Genetics. A Laboratory Manual and Handbook for Escherichia coli and Related Bacteria. Cold Spring Harbor, NY: Cold Spring Harbor Laboratory.
Miller, D. A., Luo, L., Hillson, N., Keating, T. A. \& Walsh, C. T. (2002). Yersiniabactin synthetase: a four-protein assembly line producing the nonribosomal peptide/polyketide hybrid siderophore of Yersinia pestis. Chem Biol 9, 333-344.

Miller, M. C., Parkin, S., Fetherston, J. D., Perry, R. D. \& DeMoll, E. (2006). Crystal structure of ferric-yersiniabactin, a virulence factor of Yersinia pestis. J Inorg Biochem 100, 1495-1500.

Pelludat, C., Brem, D. \& Heesemann, J. (2003). Irp9, encoded by the high-pathogenicity island of Yersinia enterocolitica, is able to convert chorismate into salicylate, the precursor of the siderophore yersiniabactin. J Bacteriol 185, 5648-5653.

Perry, R. D. (2004). Yersinia. In Iron Transport in Bacteria, pp. 219240. Edited by J. H. Crosa, A. R. Mey \& S. M. Payne. Washington, DC: American Society for Microbiology.

Perry, R. D. \& Fetherston, J. D. (1997). Yersinia pestis - etiologic agent of plague. Clin Microbiol Rev 10, 35-66.

Perry, R. D. \& Fetherston, J. D. (2004). Iron and heme uptake systems. In Yersinia Molecular and Cellular Biology, pp. 257-283. Edited by E. Carniel \& B. J. Hinnebusch. Wymondham, UK: Horizon Bioscience.

Perry, R. D., Pendrak, M. L. \& Schuetze, P. (1990). Identification and cloning of a hemin storage locus involved in the pigmentation phenotype of Yersinia pestis. J Bacteriol 172, 5929-5937.

Perry, R. D., Straley, S. C., Fetherston, J. D., Rose, D. J., Gregor, J. \& Blattner, F. R. (1998). DNA sequencing and analysis of the low-Ca ${ }^{2+}$ response plasmid pCD1 of Yersinia pestis KIM5. Infect Immun 66, 4611-4623.

Perry, R. D., Balbo, P. B., Jones, H. A., Fetherston, J. D. \& DeMoll, E. (1999). Yersiniabactin from Yersinia pestis: biochemical characterization of the siderophore and its role in iron transport and regulation. Microbiology 145, 1181-1190.

Perry, R. D., Bearden, S. W. \& Fetherston, J. D. (2001). Iron and heme acquisition and storage systems of Yersinia pestis. Recent Res Dev Microbiol 5, 13-27.

Perry, R. D., Abney, J., Mier, I., Jr, Lee, Y., Bearden, S. W. \& Fetherston, J. D. (2003a). Regulation of the Yersinia pestis Yfe and Ybt iron transport systems. Adv Exp Med Biol 529, 275-283.

Perry, R. D., Shah, J., Bearden, S. W., Thompson, J. M. \& Fetherston, J. D. (2003b). Yersinia pestis TonB: role in iron, heme and hemoprotein utilization. Infect Immun 71, 4159-4162.

Perry, R. D., Bobrov, A. G., Kirillina, O., Jones, H. A., Pedersen, L. L., Abney, J. \& Fetherston, J. D. (2004). Temperature regulation of the hemin storage $\left(\mathrm{Hms}^{+}\right)$phenotype of Yersinia pestis is posttranscriptional. J Bacteriol 186, 1638-1647.

Schaible, U. E. \& Kaufmann, S. H. E. (2004). Iron and microbial infection. Nat Rev Microbiol 2, 946-953.

Schneider, A. \& Marahiel, M. A. (1998). Genetic evidence for a role of thioesterase domains, integrated in or associated with peptide synthetases, in non-ribosomal peptide biosynthesis in Bacillus subtilis. Arch Microbiol 169, 404-410.

Staggs, T. M., Fetherston, J. D. \& Perry, R. D. (1994). Pleiotropic effects of a Yersinia pestis fur mutation. J Bacteriol 176, 76147624.

Suo, Z., Walsh, C. T. \& Miller, D. A. (1999). Tandem heterocyclization activity of the multidomain $230 \mathrm{kDa}$ HMWP2 subunit of Yersinia pestis yersiniabactin synthetase: interaction of the 1-1382 and 13832035 fragments. Biochemistry 38, 14023-14035.

Surgalla, M. J. \& Beesley, E. D. (1969). Congo red-agar plating medium for detecting pigmentation in Pasteurella pestis. Appl Microbiol 18, 834-837.

Towbin, H., Staehelin, T. \& Gordon, J. (1979). Electrophoretic transfer of proteins from polyacrylamide gels to nitrocellulose sheets: 
procedure and some applications. Proc Natl Acad Sci U S A 76, 43504354.

Venturi, V., Weisbeek, P. \& Koster, M. (1995). Gene regulation of siderophore-mediated iron acquisition in Pseudomonas: not only the Fur repressor. Mol Microbiol 17, 603-610.
Wang, R. F. \& Kushner, S. R. (1991). Construction of versatile lowcopy-number vectors for cloning, sequencing and gene expression in Escherichia coli. Gene 100, 195-199.

Edited by: P. C. F. Oyston 